\title{
Newly Developed Endotoxin Measurement Method (the Endotoxin Activity Assay) May Reflect the Severity of Sepsis
}

\author{
Kiyohide Ishihata ${ }^{1,2}$, Yasuyuki Kakihana ${ }^{1}$, Tomotsugu Yasuda $^{1}$, Tohru Imabayashi ${ }^{3}$, \\ Norifumi Nakamura ${ }^{2}$ \\ ${ }^{1}$ Department of Emergency and Intensive Care Medicine, Faculty of Medicine, Kagoshima University, Kagoshima, Japan; ${ }^{2}$ Depart- \\ ment of Oral and Maxillofacial Surgery, Field of Oral and Maxillofacial Rehabilitation, Advanced Therapeutic Course, Kagoshima \\ University Graduate School of Medical and Dental Sciences, Kagoshima, Japan; ${ }^{3}$ Department of Anesthesiology, Faculty of Medi- \\ cine, Kagoshima University, Kagoshima, Japan. \\ Email: ishihata@dent.kagoshima-u.ac.jp
}

Received October $29^{\text {th }}, 2012$; revised November $30^{\text {th }}, 2012$; accepted December $15^{\text {th }}, 2012$

\begin{abstract}
Objectives: Endotoxin (ET) is a structural molecule of the Gram-negative bacilli extracellular membrance, which activates targeted cells including macrophages and neutrophils, and causes septic shock. But it is known that the conventional ET measurement method has many problems; for example, a discrepancy between plasma ET concentration and clinical manifestration in the septic patients has been reported. The purpose of this study was to evaluate the usefulness of a newly developed method (Endotoxin Activity Assay (EAA)) to measure the ET activity (EA) in patients under sepsis compared with the prior method of the limulus amebocyte lysate (LAL) assay and explore the association between EA levels and patients' severity. Method: We measured the EA levels in 40 patients (aged $63.5+/-17.7$ years) admitted to the ICU. EA level was measured using a chemiluminometer (Autolumat LB953; EG \& Berthold). Patients were divided into 5 groups: 1) control group; 2) systemic inflammatory response syndrome (SIRS) group; 3) sepsis (SIRS and infection) group; 4) severe sepsis group and 5) septic shock group. We then compared the EA level between each group and control group. We made the statistical evaluation by unpaired $t$ test and significant difference was $\mathrm{p}<0.05$. Results: The EA levels were significantly increased as sepsis severity rises. The measured EA levels were $(0.18+/-$ $0.09),(0.33+/-0.19),(0.39+/-0.16),(0.65+/-0.25)$ and $(0.78+/-0.34)$ in control, SIRS, sepsis, severe sepsis and septic shock groups, respectively. In the EA level measured by EAA, severe patients had a tendency to exceed the cutoff value. Conclusion: The EA levels were significantly correlated as patients' severity rise. Measuring EA levels on admittion to ICU may provide a mechanism to identify and target severe septic patients.
\end{abstract}

Keywords: Endotoxin Activity Assay; Endotoxin; Sepsis

\section{Introduction}

Endotoxin (ET), a complex lipopolysaccharide (LPS) that is present in the cell walls of Gram-negative bacteria, is a potent trigger of innate immunity [1]. Isolated and purified from the wall of several gram-negative bacteria, it has been used to investigate many aspects of the immuno-inflammatory response of sepsis through inoculation in the laboratory animal or in humans [2]. ET was first identified in the serum of patients undergoing cardiopulmonary bypass (CPB) over 20 years ago and proposed as a potential mediator of multiple organ failure and prolonged recovery after cardiac surgery $[3,4]$. ET activates the targeted cells such as macrophages and neutrophils, and is the most important factor in the originating materials of sepsis and septic shock. Endo- toxemia has been detected in a variety of disorders, including sepsis, liver disease, and vascular disease, and in patients who have sustained trauma or who have undergone cardiopulmonary bypass [5-11].

Measuring the concentration of ET in human disease has been notoriously difficult. The commonly used method, the chromogenic limulus amebocyte lysate (LAL) assay, is based on the ability of ET to induce coagulation of hemolymph in the horseshoe crab, limulus polyphemus. The utility of this assay has been limited because of circulating inhibitors of the coagulation reaction [12]. The question of specificity of LAL assay in the diagnosis of endotoxemia has been a limiting factor of its clinical application $[13,14]$. In recent years, a novel assay for ET, based on the ability of antigen-antibody complex to prime neutrophils and augmented respiratory burst re- 
sponse (Endotoxin Activity Assay: EAA) has been developed. EAA is simple and more accurate than the LAL assay, since then, EAA has been applied in a clinical setting and the assay's efficacy has been recognized $[1,15]$

It is indispensable to measure the ET activity (EA level) in blood for diagnosing the sepsis and septic shock at the early stage and to begin appropriate treatment.

Selecting a Template (Sub-Heading 2.1). First, confirm that you have the correct template for your paper size. This template has been tailored for output on the custom paper size $(21 \mathrm{~cm} \times 28.5 \mathrm{~cm})$.

\section{Materials and Methods}

\subsection{Patients Characteristics (Table 1)}

This study protocol was approved by the Research Ethic Board of Kagoshima University Hospital and all patients provided informed consent. A totaled 40 adults patients admitted to the ICU in our institution were enrolled for the study from November 2006 to July 2007. On admission and at least one time on the next day, clinical history and laboratory data were taken, and total patients measured EA levels were 91 patients. The patients were divided into 5 groups in according to patients' general condition: 1) control group: 12 patients, those who were preoperative status; 2) SIRS group: 17 patients, Systemic Inflammatory Response Syndrome (SIRS) was considered to be present at least 2 of 3 criteria were met: temperature above $38^{\circ} \mathrm{C}$ or below $36^{\circ} \mathrm{C}$, heart rate of more than 90 beats $/ \mathrm{min}$, respiratory rate of more than 20 breasts/min or partial pressure of carbon dioxide of less than $32 \mathrm{mmHg}$, or white blood cell count above 12,000 $\mathrm{mm}^{3}$ or below $4000 \mathrm{~mm}^{3}$ [16]; 3) sepsis group: 14 patients, those who were diagnosed with sepsis, episodes of infection were diagnosed by microbiologic, laboratory, radiologic and operative data; 4 ) severe sepsis group: 32 patients and 5) septic shock group: 16 patients. Severe sepsis and septic shock were defined according to the consensus definition of the American College of Chest Physicians/Society of Critical Care Medicine Consensus Conference Committee [16]. On admission, blood samples were withdrawn for Endotoxin Activity (EA) assay and during ICU stay blood and other laboratory data were collected at least one time a day. Blood for ET assay was collected in ET free tube, with EDTA as an anticoagulant.

\subsection{Endotoxin Activity Assay (EAA)}

The EAA is described in detail elsewhere $[1,2,5,17]$. The EAA is a rapid chemiluminescent immunodiagnostic test kit that contains labeled glass tubes of specific reagents and a container of liquid substrate. The EAA is approved by Food and Drug Administration (FDA) as a method to evaluate ET activity (EA) in whole blood because of its' high sensitivity and specificity for LPS and has demonstrated a strong clinical signal with a patient's clinical status $[5,18]$. The EAA measures the EA in whole blood by the priming of host neutrophil respiratory burst activeity via complement opsonized LPS-IgM complexes. The luminol reaction in the presence of immune complexes emits light energy. This light energy is measured and recorded by a luminometer. The Relative Light Units (RLU) measured by the instruments is converted by a calculation into an Endotoxin Activity (EA) level which is reported as a percentage of the total possible activity $[2,17]$. The measured EA levels were divided into the following 3 groups: low (EA level $<0.4$ ), intermediate (EA level 0.4 to $<0.6$ ) and high (EA level $\geq 0.6$ ).

\subsection{Limulus Amoebocyte Lysate (LAL) Assay}

Determination of endotoxin using the LAL technique was conducted with the Toxinometer MT-358, Endotoxintest Wako and Beta-Gulcan Test Wako (Wako Pure Chemical Industries, Ltd., Osaka, Japan). The principle of the test is based on the fact that bacteria cause intravascular coagulation in the American horseshoe crab, Limulus polyphemus. The agent responsible for the clotting phenomena resided in the crab's amoebocytes, or circulating blood cells, and that pyrogen (bacterial endotoxin) triggered the turbidity and gel-forming reaction enzymatically. Thus, ET causes an opacity and gelation in limulus amoebocyte assay, which is based on an enzymatic reaction.

The chromogenic limulus amebocyte lysate assay has been most widely used to detect ET contamination of drugs and fluids; however, its utility in biological samples has been limited, because of circulating inhibitors of the coagulation reaction. Moreover, other microbial products, notably from fungi, can activate the limulus reaction, so the assay is not specific for ET.

\subsection{Statistical Analysis}

Unpaired $t$ test was used to compare the EA level between each group and control group. Comparison between Endotoxin Activity Assay and LAL method was evaluated using paired $t$ test. Normally distributed data are presented as mean $+/-\mathrm{SD}$. Statistical significant was assumed for values $\mathrm{p}<0.05$.

\section{Results}

A total of 91 patients were recruited for the study. All patients were divided into 5 groups: 1) control group; 2) SIRS group; 3) sepsis group; 4) severe sepsis group and 5) septic shock group based on their clinical findings 
(Table 1) and had their EA level measured on admission day and at least one time a day during the course of the ICU stay. The EA levels were significantly increased as sepsis severity rise. The measured EA levels were 0.18 $+/-0.09,0.33+/-0.19,0.39+/-0.16,0.65+/-0.25$ and $0.78+-0.34$ in control, SIRS, sepsis, severe sepsis and septic shock group, respectively (Figure 1). In the LAL method, 4 of the severe sepsis group and 2 of the septic shock group exceeded the cutoff value. In the EA level, severe patients had a tendency to exceed the cutoff value. There was no significant correlation between the EA levels and ET values measured by LAL assay (Figure 2). Patients with Gram-negative infection and Gram-positive infection had higher mean EA levels measured by EAA than did those control group $(p<0.05)$; no significant difference between Gram-negative infection and Grampositive infection (Figure 3).

Table 1. Patients characteristics $(n=91)$. Characteristics of the study population.

\begin{tabular}{ccc}
\hline 1. Control group & Preoperative status & 12 \\
\hline & $\begin{array}{c}\text { Temperature above } 38^{\circ} \mathrm{C} \text { or below } 36^{\circ} \mathrm{C}, \\
\text { heart rate of more than } 90 \text { beats } / \mathrm{min}, \\
\text { respiratory rate of more than } 20 \text { breasts } / \mathrm{min} \\
\text { or partial pressure of carbon dioxide of less } \\
\text { than } 32 \mathrm{mmHg} \text {, white blood cell count } \\
\text { above } 12,000 \mathrm{~mm}^{3} \text { or below } 4000 \mathrm{~mm}^{3} \text { at } \\
\text { least two of these criteria met. }\end{array}$ & 17 \\
3. Sepsis group & SIRS and infection. & 14 \\
4. Severe sepsis group & Sepsis and multiple organ failure. & 32 \\
5. Septic shock group & $\begin{array}{l}\text { Severe sepsis and intractable hypotension. } \\
\text { She }\end{array}$ & 16
\end{tabular}

SIRS: Systemic Inflammatory Response Syndrome.

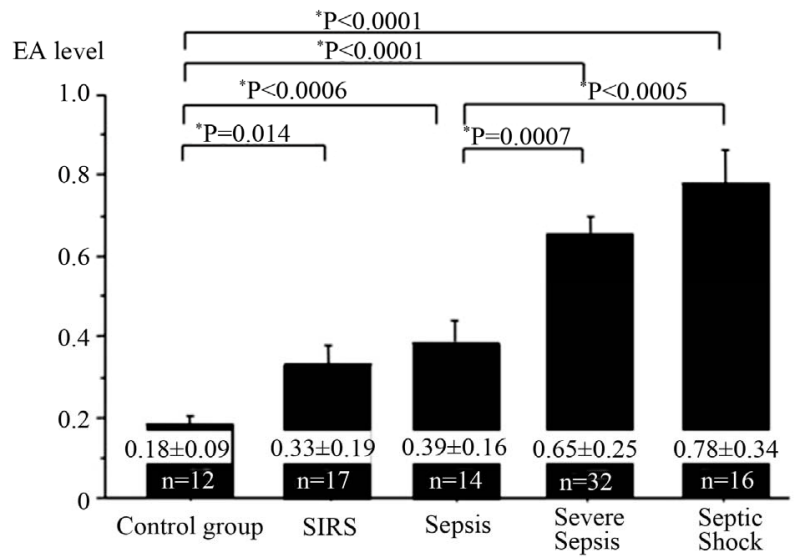

Figure 1. Endotoxin Activity Assay measureing. Endotoxin Activity (EA) levels were significantly elevated as severity of illness increased. The severe sepsis group and septic shock group showed the high Endotoxin Activity (EA) level (severe sepsis group: $0.65+/-0.25$, septic shock group: 0.78 $+/-$ 0.34). Endotoxin Activity (EA) levels are expressed as mean \pm standard deviation.

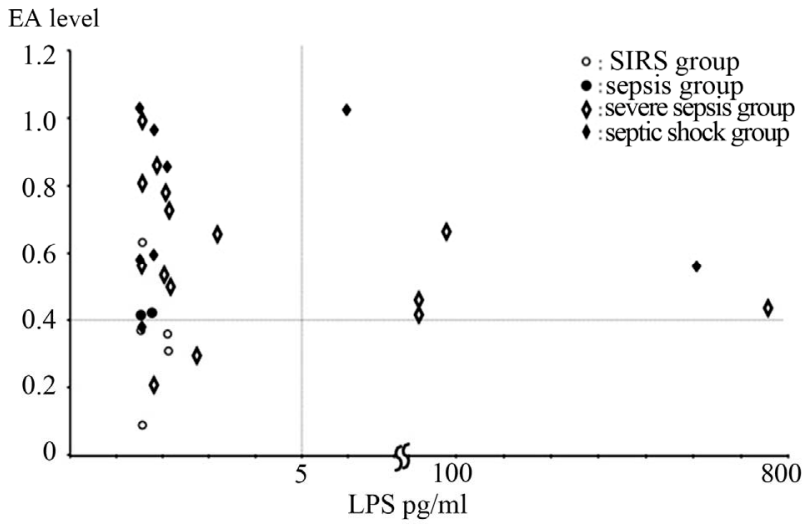

Figure 2. Correlation with Endotoxin Activity Assay (EAA) and limulus amebocyte lysate (LAL) assay. Correlation significant between EAA and LAL did not accept. O: SIRS group; •: sepsis group; $\diamond$ : severe sepsis group; $\bullet$ : septic shock group. There was no significant correlation between the EA levels and ET values measured by LAL assay.

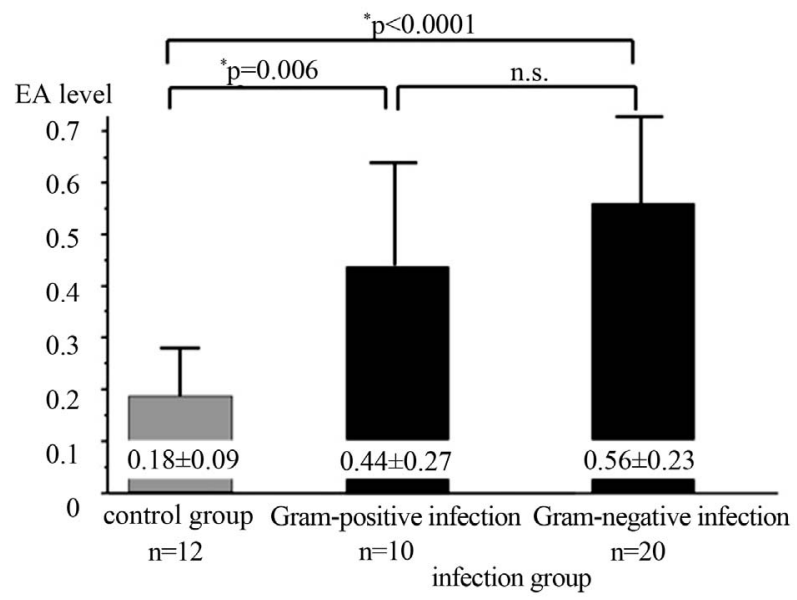

Figure 3. Comparison of the Endotoxin Activity (EA) level according to causing bacteria. The Gram-negative-bacteria infection group and the Gram-positive-bacteria infection group were significantly high Endotoxin Activity (EA) levels than a control group. There was no significant difference between a Gram-negative-bacteria infection group and a Gram-positive-bacteria infection group. Endotoxin Activity (EA) levels are expressed as mean \pm standard deviation.

\section{Discussion}

Endotoxin (ET), a complex lipopolysaccharide (LPS), is present in the cell wall of Gram-negative bacteria and is capable of stimulating the immune system and coagulation cascade $[1,18]$.

A distinct carrier protein, LPS-binding protein, transports circulating ET and facilitates its recognition by the cell through a unique receptor, CD14; an associated pattern-recognition receptor, TLR4; and an accessory protein, MD2; that transduces the ET signal to the cell nucleus, leading to the expression of a complex network on 
inflammatory mediator [1]. As a result, cytokines such as TNF and IL-6 are induced, patients occurs systemic inflammatory response syndrome (SIRS), sepsis and furthermore septic shock. Failure in early detection of sepsis causes multiple organ failure and results in high mortality. High levels of Endotoxin Activity are associated with worse clinical outcomes $[18,19]$. For this reason, the diagnosis of the sepsis must be given immediately and it is necessary to intervene it at the early stage of treatment. However, recent years, effectiveness of measurement of ET values is still controversial.

In the past, only widely available method for measuring ET value has been the LAL assay; however, its use to detect and quantify ET in plasma or whole blood has been problematic. Its utility in biological sample has been limited because of circulating inhibitors of the coagulation reaction. In addition, other microbial products, notably from fungi, can activate the LAL reaction, so the assay is not specific for ET $[5,12,20,21]$. The EAA detects ET as the priming of the patient's own neutrophils by complexs of ET and specific antiendotoxin antibody; it is thus more sensitive and more specific to Gramnegative bacterial ET than the LAL assay is $[5,17]$. It can be performed using as little as $100 \mu \mathrm{l}$ whole blood and results are available within $30 \mathrm{~min}$. Despite a problem that the absolute value of the amount of ET cannot be requested like the LAL assay, because the EAA measures ET value by the chemiluminescence, EAA is capable of rapid and high-sensitive detection compared to the LAL method; therefore, in sepsis, which is a clinical state that requires prompt action, it plays an important role in diagnosis and treatment [15]. We compared results obtained with a new, rapid, assay for ET (EAA) with those obtained using the chromogenic LAL assay in a group of patients admitted to an ICU. In the severe sepsis group and septic shock group, 6 patients had ET values greater than the cut off value $(5 \mathrm{pg} / \mathrm{ml})$ at the time of ICU admission, by using LAL assay. When samples were assayed using the EAA; no such correlation was found when samples were assayed using LAL methods. Studies comparing EAA and LAL show considerable variability in the prevalence of endotoxemia and no such correlation were found when samples were assayed using the EAA and LAL $[5,12]$. There is no standard method for measuring ET value in blood now. For this reason, a further examination is necessary which the EAA or the LAL assay is an appropriate method to measure ET value in blood.

Endotoxemia may arise from two sources: 1) Gramnegative infection; or 2) Translocation of bacterial products from the gastrointestinal tract $[5,18,22]$. This study showed that both Gram-positive infection cases and Gram-negative infection cases demonstrated significant high EA levels (Figure 3). Some previous studies showed same results and Abdala et al. suggested that the presence of endotoxemia was unaccompanied by bacteremia and the possible etiology of endotoxemia was intestinal translocation with impairment of hepatic clearance and was therefore not related to Gram-negative bacteremia $[5,6,18,23]$. Therefore, identification of measureing EA level using EAA of Gram negative-bacteria infection needs the further examination.

Endotoxemia has been reported to be common in patients with sepsis, chronic liver disease with cirrhosis and leukaemia or lymphoma, as well as in patients undergoing cardiopulmonary bypass (CPB) $[5,19,24]$. Endotoxaemia correlates with mortality in patients with meningococcemia, severe pancreatitis and extensive burn injury $[5,25,26]$. In this study, we examined the relation between the EA level and patients' severity. We revealed that the EA levels seem increased as the patients' severity rise. Valenza et al. have described that patients with high EA levels had a longer ICU length of stay and trend towards longer hospital length stay, some patients with high EA levels on admission, demonstrated that white blood cell count, SIRS criteria, and lactate did not significantly decrease as the following day after admission [2]. However some patients had higher EA levels than normal levels of ET despite their low level of complexity (APACHE II score). Whether this was indicative of an ongoing inflammatory process or adequacy of perfusion is difficult to determine. A previous study suggested that measurement of EA level using EAA is a potential tool to stratify patients to more aggressive care or to allocate resources in dynamic ICUs recruiting post-operative patients for routine monitoring [2]. In addition, Klein et al. suggested that, using EAA, endotoxemia occurs in some patients having cardiac surgery, although rarely at high levels [4]. Furthermore, endotoxemia at the conclusion of cardiopulmonary bypass (CPB) is reported to be associated with a significant risk of the development of postoperative infections [26]. It has been described that translocation of ET through gut or lung tissue takes part in the endotoxaemia in the critically ill patients and during CPB [5,4,26]. Patients with high EA level have been found to have an increased risk of infection or high mortality. Nevertheless, the mechanism why critical ill patients have high EA level cannot be elucidated and has been hypothesized that this phenomenon may be linked to translocation of ET.

We revealed that critical ill patients under severe sepsis or septic shock had higher EA levels significantly. Patients who had intermediate or high levels of ET measured by EAA on the day of admission were clearly a sicker population, as reflected in higher admission APACHEII scores and a greater prevalence of severe 
sepsis. Moreover, patients with the highest levels of circulating ET had a significantly increased risk of mortality while in the ICU. Thus, the presence of endotoxemia identifies a high-risk subpopulation of critically ill patients [1]. Therefore, we considered that the measuring EA levels using EAA might be a useful for evaluating patients' severity and might be a useful parameter to start treatment at the early stage. The availability of a relatively simple bedside assay for blood ET levels may identify a high-risk population of critically ill patients who may benefit from adjunctive therapy [12].

There are limitations to our study. We studied a relatively small number of patients for determination the clinical condition such as severe sepsis or septic shock and the lack of intensive follow-up data. As discussed above, the mechanism underlying the correlation of higher EA level and patients' severity is unknown. Further studies are needed to elucidate why severe patients have high EA levels.

\section{Conclusion}

In this study we have investigated the prevalence of endotoxemia of patients admitted to an ICU by measureing EA level using EAA. The EA levels were significantly increased as sepsis severity rise. High EA levels were associated with a grade of severity of patients. Further study is needed to determine the clinical usefulness of EAA in critical ill patients.

\section{REFERENCES}

[1] J. C. Marshall, D. Foster, J.-L. Vincent, D. J. Cook, J. Cohen, R. P. Dellinger, S. Opal, E. Abraham, S. J. Brett, T. Smith, S. Mehta, A. Derzko, A. Romaschin, MEDIC Study, "Diagnostic and Prognostic Implications of Endotoxemia in Critical Illness: Results of the MEDIC Study," Journal of Infectious Diseases, Vol. 190, No. 3, 2004, pp. 527-534. doi:10.1086/422254

[2] F. Valenza, L. Fagnani, S. Coppola, S. Froio, F. Sacconi, C. Tedesco, M. Maffioletti, M. Pizzocri, V. Salice, M. L. Ranzi, C. Marenghi and L. Gattinoni, "Prevalence of Endotoxemia after Surgery and Its Association with ICU Length of Stay," Critical Care, Vol. 13, No. 3, 2009, p. R102. doi:10.1186/cc7934

[3] D. A. Rocke, S. L. Gaffin, M. T. Wells, Y. Koen and J. G. Brock-Utine, "Endotoxemia Associated with Cardiopulmonary Bypass," Journal of Thoracic Cardiovascular Surgery, Vol. 93, No. 6, 1987, pp. 832-837.

[4] D. J. Klein, F. Briet, R. Nisenbaum, A. D. Romaschin and C. D. Mazer, "Endotoxemia Related to Cardiopulmonary Bypass Is Associated with Increased Risk of Infection after Cardiac Surgery: A Prospective Observational Study," Critical Care, Vol. 15, No. 1, 2011, p. R69. doi:10.1186/cc10051

[5] J. C. Marshall, P. M. Walker, D. M. Foster, D. Harris, M.
Ribeiro, J. Paice, A. D. Romaschin and A. N. Derzko, "Measurement of Endotoxin Activity in Critically Ill Patients Using Whole Blood Neutrophil Dependent Chemiluminescence," Critical Care, Vol. 6, No. 4, 2002, pp. 342-348. doi:10.1186/cc1522

[6] R. Danner, R. Elin, J. Hosseini, R. Wesley, J. Reilly and J. Parillo, "Endotoxemia in Human Septic Shock," Chest, Vol. 99, No. 1, 1991, pp. 169-175.

doi:10.1378/chest.99.1.169

[7] L. C. Casey, R. A. Balk and R. C. Bone, "Plasma Cytokine and Endotoxin Levels Correlate with Survival in Patients with the Sepsis Syndrome," Annals Internal Medicine, Vol. 119, No. 8, 1993, pp. 771-778.

[8] C. H. Wortel, M. A. von der Möhlen, S. J. van Deventer, C. L. Sprung, M. Jastremski, M. J. Lubbers, C. R. Smith, I. E. Allen and J. W. ten Cate, "Effectiveness of a Human Monoclonal Anti-Endotoxin Antibody (HA-1A) in GramNegative Sepsis: Relationship to Endotoxin and Cytokine Levels," Journal of Infectious Diseases, Vol. 166, No. 6 , 1992, pp. 1367-1374. doi:10.1093/infdis/166.6.1367

[9] J. Bion, I. Badger and H. Crosby, "Selective Decontamination of the Digestive Tract Reduces Gram-Negative Pulmonary Colonization but Not Systemic Endotoxemia in Patients Undergoing Elective Liver Transplantation," Critical Care Medicine, Vol. 22, No. 1, 1994, pp. 40-49.

[10] G. B. Gaeta, P. Perna, L. E. Adinolfi, R. Utili and G. Ruggiero, "Endotoxemia in a Series of 104 Patients with Chronic Liver Diseases: Prevalence and Significance," Digestion, Vol. 23, No. 4, 1982, pp. 239-244.

[11] R. Roumen, J. Frieling and H. Van Tits, "Endotoxemia after Major Vascular Operations," Journal of Vascular Surgery, Vol. 18, No. 5, 1993, pp. 853-857. doi:10.1016/0741-5214(93)90341-I

[12] S. Dholakia, D. Inwald, H. Betts and S. Nadel, "Endotoxemia in Pediatric Critical Illness-A Pilot Study," Critical Care, Vol. 15, No. 3, 2011, p. R141. doi:10.1186/cc10264

[13] P. C. Fink, L. Lehr, R. M. Urbaschek and J. Kozak, "Limulus Amebocyte Lysate Test for Endotoxemia: Investigations with a Femtogram Sensitive Spectrophotometric Assay," Klinische Wochenschrift, Vol. 59, No. 5, 1981, pp. 213-218. doi:10.1007/BF01476578

[14] M. Sakata, T. Inoue, M. Todokoro and M. Kunitake, "Limulus Amebocyte Lysate Assay for Endotoxins by an Adsorption Method with Polycation-Immobilized Cellulose Beads," Analytical Sciences, Vol. 26, No. 3, 2010, pp. 291-296. doi:10.2116/analsci.26.291

[15] Y. Sanada, K. Mizuta, T. Urahashi, Y. Ihara, T. Wakiya, N. Okada, N. Yamada, K. Ushijima, S. Otomo, K. Sakamoto and Y. Yasuda, "Impact of Hepatic Clearance of Endotoxin Using Endotoxin Activity Assay," Hepatology International, Vol. 6, 2011, pp. 489-493. doi:10.1007/s12072-011-9289-4

[16] R. C. Bone, R. A. Balk, F. B. Cerra, R. P. Dellinger, A. M. Fein, W. A. Knaus, R. M. Schein and W. J. Sibbald, "Definitions for Sepsis and Organ Failure and Guidelines for the Use of Innovative Therapies in Sepsis. The ACCP/ SCCM Consensus Conference Committee. American Col- 
lege of Chest Physicians/Society of Critical Care Medicine," Chest, Vol. 101, No. 6, 1992, pp. 1644-1655. doi:10.1378/chest.101.6.1644

[17] A. D. Romaschin, D. M. Harris, M. B. Ribeiro, J. Paice, D. M. Foster, P. M. Walker and J. C. Marshall, "A Rapid Assay of Endotoxin in Whole Blood Using Autologous Neutrophil Dependent Chemiluminescence," Journal of Immunological Methods, Vol. 212, No. 2, 1998, pp. 169185. doi:10.1016/S0022-1759(98)00003-9

[18] I. Hilmi, J. A. Kellum, R. Planinsic, D. Foster, A. Abdullah, D. Damian, S. Gligor, D. Klein and K. Abu-Elmagd, "Endotoxemia is Common Following Abdominal Organ Transplantation and Is Associated with Reperfusion and Rejection," Journal of Organ Dysfunction, Vol. 5, No. 4, 2009, pp. 254-260. doi:10.3109/17471060902859113

[19] P. G. Jansen, H. Te Velthuis, H. M. Oudemans-Van Straaten, E. R. Bulder, S. J. van Deventer, A. Sturk, L. Eijsman and C. R. Wildevuur, "Perfusion-Related Factors of Endotoxin Release during Cardiopulmonary Bypass," European Journal Cardiothoracic Surgery, Vol. 8, No. 3, 1994, pp. 125-129. doi:10.1016/1010-7940(94)90167-8

[20] R. J. Elin, R. A. Robinson, A. S. Levine and S. M. Wolff, "Lack of Clinical Usefulness of the Limulus Test in the Diagnosis of Endotoxemia," New England Journal of Medicine, Vol. 293, No. 11, 1975, pp. 521-524. doi:10.1056/NEJM197509112931102

[21] D. W. Bates, J. Parsonnet, P. A. Ketchum, E. B. Miller, T. J. Novitsky, K. Sands, P. L. Hibberd, P. S. Graman, P. N. Lanken, J. S. Schwartz, K. Kahn, D. R. Snydman, R. Moore, E. Black and R. Platt, "Limulus Amebocyte Lysate Assay for Detection of Endotoxin in Patients with Sepsis Syndrome," Clinical Infectious Diseases, Vol. 27, No. 3, 1998, pp. 582-591. doi:10.1086/514713

[22] M. Kjelgaard-Hansen, B. Wiinberg, B. Aalbaek, L. Olsen,
D. Harris, A. Romaschin, A. T. Kristensen and A. L. Jensen, "Endotoxin Activity in Whole Blood Measured by Neutrophil Chemiluminescence Is Applicable to Canine Whole Blood," Comparative Immunology, Microbiology and Infectious Diseases, Vol. 31, No. 6, 2008, pp. 477485. doi:10.1016/j.cimid.2007.08.004

[23] E. Abdala, C. E. S. Baía, S. Mies, P. C. B. Massarollo, N. de Paula Cavalheiro, V. R. M. Baía, C. A. F. Inácio, H. C. Sef and A. A. Barone, "Bacterial Translocation during Liver Transplantation: A Randomized Trial Comparing Conventional with Venovenous Bypass vs. Piggyback Methods," Liver Transplantation, Vol. 13, No. 4, 2007, pp. 488-496. doi:10.1002/1t.21085

[24] G. Behre, I. Schedel, B. Nentwig, B. Wörmann, M. Essink and W. Hiddemann, "Endotoxin Concentration in Neutropenic Patients with Suspected Gram-Negative Sepsis: Correlation with Clinical Outcome and Determination of Anti-Endotoxin Core Antibodies during Therapy with Polyclonal Immunoglobulin M-Enriched Immunoglobulins," Antimicrobial Agents and Chemotherapy, Vol. 36, No. 10, 1992, pp. 2139-2146. doi:10.1128/AAC.36.10.2139

[25] P. Brandtzaeg, P. Kierulf, P. Gaustad, A. Skulberg, J. N. Bruun, S. Halvorsen and E. Sørensen, "Plasma Endotoxin as a Predictor of Multiple Organ Failure and Death in Systemic Meningococcal Disease," Journal of Infectious Diseases, Vol. 159, No. 2, 1989, pp. 195-204. doi:10.1093/infdis/159.2.195

[26] N. B. Aydin, H. Gercekoglu, B. Aksu, V. Ozkul, T. Sener, I. Kiygil, T. Turkoglu, S. Cimen, F. Babacan and M. Demirtas, "Endotoxemia in Coronary Artery Bypass Surgery: A Comparison of the Off-Pump Technique and Conventional Cardiopulmonary Bypass," Journal of Thoracic and Cardiovascular Surgery, Vol. 125, No. 4, 2003, pp. 843-848. doi: $10.1067 / \mathrm{mtc} .2003 .323$ 